\title{
Digital Implementation of Artificial Neural Network for Function Approximation and Pressure Control Applications
}

\author{
Sangeetha $\mathrm{T}^{\# 1}$, Meenal $\mathrm{C}^{\# 2}$ \\ ${ }^{1,2}$ Department of Electronics and Communication Engineering \\ ${ }^{1}$ PG Scholar, Mount Zion College of Engineering and Technology, Pudukkottai, Tamil Nadu, India. \\ ${ }^{2}$ Assistant Professor, Mount Zion College of Engineering \& Technology, Pudukkottai, TamilNadu India
}

\begin{abstract}
The soft computing algorithms are being nowadays used for various multi input multi output complicated non linear control applications. This paper presented the development and implementation of back propagation of multilayer perceptron architecture developed in FPGA using VHDL. The usage of the FPGA (Field Programmable Gate Array) for neural network implementation provides flexibility in programmable systems. For the neural network based instrument prototype in real time application. The conventional specific VLSI neural chip design suffers the limitation in time and cost. With low precision artificial neural network design, FPGA have higher speed and smaller size for real time application than the VLSI design. The challenges are finding an architecture that minimizes the hardware cost, maximizing the performance, accuracy. The goal of this work is to realize the hardware implementation of neural network using FPGA. Digital system architecture is presented using Very High Speed Integrated Circuits Hardware Description Language (VHDL)and is implemented in FPGA chip. MATLAB ANN programming and tools are used for training the ANN. The trained weights are stored in different RAM, and is implemented in FPGA. The design was tested on a FPGA demo board.
\end{abstract}

Keywords- Backpropagation, field programmable gate array (FPGA) hardware implementation, multilayer perceptron, pressure sensor, Xilinx FPGA.

\section{Introduction}

Implementation of ANNs falls into two categories: Software implementation and hardware implementation. ANNs are implemented in software, and are trained and simulated on general-purpose sequential computers for emulating a wide range of neural networks models. Software implementations offer flexibility. However hardware implementations are essential for applicability and for taking the advantage of ANN's inherent parallelism. Specific-purpose fixed hardware implementations(i.e. VLSI) are dedicated to a specific ANN model. VLSI implementations of ANNs provide high speed in real time applications and compactness. However, they lackflexibility for structural modification and are prohibitively costly.

Software implementations can be quickly constructed, adapted, and tested for a wide range of applications. However, in some cases, the use of hardware architectures matching the parallel structure of ANNs is desirable to optimize performance or reduce the cost of the implementation, particularly for applications demanding high performance. Unfortunately, hardware platforms suffer from several unique disadvantages such as difficulties in achieving high data precision with relation to hardware cost, the high hardware cost of the necessary calculations, and the inflexibility of the platform as compared to software.

In this work, aimed address some of these disadvantages by developing and implementing a field programmable gate array (FPGA)-based architecture of a neural network with learning capability. Exploiting the reconfigurability of FPGAs, we are able to perform fast prototyping of hardware-based ANNs to find optimal application specific configurations. In particular, the ability to quickly generate a range of hardware configurations gives us the ability to perform a rapid design space exploration navigating the cost/speed/accuracy tradeoffs affecting hardware-based ANNs.

\section{ARTIFICIAL NEURAL NETWORKS (Anns)}

Artificial neural networks (ANN's, or simply NN's) are inspired by biological nervous systems and consist of simple processing elements (PE, artificial neurons) that are interconnected by weighted connections. The predominantly used structure is a multilayered feed-forward network (multilayer perception),i.e., the nodes (neurons) are arranged in several layers (input layer, hidden layers, output layer), and the information flow is only between adjacent layers [4].An artificial neuron is a very simple processing unit. It calculates the weighted sum of its inputs and passes it through a nonlinear transfer function to produce its output signal.The predominantly used transfer functions are so-called "sigmoid" or "squashing" functions that compress an infinite input range to a finite output range, e.g., $[-1,+1]$. 


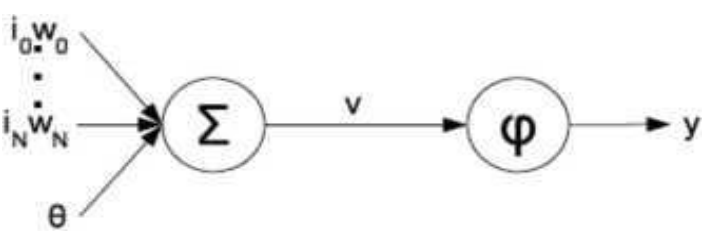

Fig.1 processing element

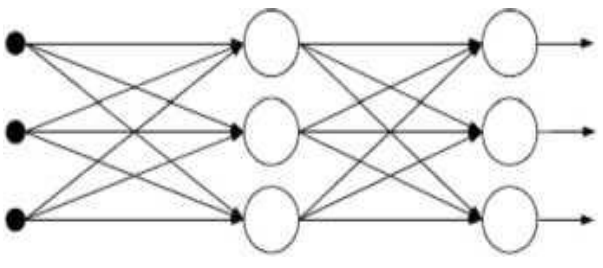

Fig.2 Multilayer perception model

Neural networks can be "trained" to solve problems that are difficult to solve by conventional computer algorithms. Training refers to an adjustment of the connection weights, based on a number of training examples that consist of specified inputs and corresponding target outputs. Training is an incremental process where after each presentation of a training example, the weights are adjusted to reduce the discrepancy between the network and the target output. Popular learning algorithms are variants of gradient descent (e.g., error-backpropagation), radial basis function adjustments [4], etc. Neural networks are well suited to a variety of nonlinear problem solving tasks. For example, tasks related to the organization, classification, and recognition of large sets of inputs.

\subsection{Multilayer Perceptrons (MLPs)}

MLPs (Fig. 2) are layered fully connected feed-forward networks. That is, all PEs (Fig. 1) in two consecutive layers are connected to one another in the forward direction.

During the network's forward pass each PE computes its output ${ }_{\mathrm{yk}}$ from the input $\boldsymbol{i}_{\boldsymbol{k}}$ it receives from each PE in the preceding layer as shown here

$$
\mathrm{yk}=\varphi k(v k)
$$

where $\varphi k$ is the squashing function of PE $\boldsymbol{k}$ whose role is to constrain the value of the local field

$$
\mathrm{vk}=\sum w k j i k j+\theta k
$$

$W k j$ is the weight of the synapse connecting neuron $\boldsymbol{k}$ to neuron $\boldsymbol{j}$ in the previous layer, and $\theta k \boldsymbol{k}$ is the bias of neuron $\boldsymbol{k}$. Equation (1) is computed sequentially by layer from the first hidden layer which receives its input from the input layer to the output layer, producing one output vector corresponding to one input vector.

The network's behavior is defined by the values of its weights and bias. It follows that in network training the weights and biases are the subjects of that training. Training is performed using the backpropagation algorithm after every forward pass of the network.

\subsection{Backpropagation Algorithm}

The backpropagation learning algorithm allows us to compute the error of a network at the output, then propagate that error backwards to the hidden layers of the network adjusting the weights of the neurons responsible for the error. The network uses the error to adjust the weights in an effort to let the output yj approach the desired output $\boldsymbol{d j}$.

Backpropagation minimizes the overall network error by calculating an error gradient for each neuron from which a weight change $\Delta$ wjiis computed for each synapse of the neuron. The error gradient is then recalculated and propagated backwards to the previous layer until weight changes have been calculated for all layers from the output to the first hidden layer.

The weight correction for a synaptic weight connecting neuron $\boldsymbol{i}$ to neuron $\boldsymbol{j}$ mandated by backpropagation is defined by the delta rule

$$
\Delta \mathrm{wji}=\eta \delta j y i
$$

where $\eta$ is the learning rate parameter, $\delta j$ is the local gradient of neuron $\boldsymbol{j}$, and $y \boldsymbol{i}$ is the output of neuron $\boldsymbol{i}$ in the previous layer.

Calculation of the error gradient can be divided into two cases: for neurons in the output layer and for neurons in the hidden layers. This is an important distinction because we must be careful to account for the effect that changing the output of one neuron will have on subsequent neurons. For output neurons, the standard definition of the local gradient applies

$$
\delta j=e j \varphi^{\prime} j(v j)
$$

For neurons in a hidden layer, we must account for the local gradients already computed for neurons in the following layers up to the output layer. The new term will replace the calculated error e since, because hidden 
neurons are not visible from outside of the network, it is impossible to calculate an error for them. So, we add a term that accounts for the previously calculated local gradients

$$
\delta j=\varphi^{\prime} j(v j) \sum \delta k w k j
$$

Where $\boldsymbol{j}$ is the hidden neuron whose new weight we are calculating, and $\boldsymbol{k}$ is an index for each neuron in the next layer connected to $j$.

As we can see from (4) and (5), we are rewe are required to differentiate the activation function $\varphi j$ with respect to its own argument, the induced local field $v j$. In order for this to be possible, the activation function must of course be differentiable. This means that we cannot use non continuous activation functions in a backpropagation-based network. Two continuous, nonlinear activation functions commonly used in back propagation networks are the sigmoid function

$$
\varphi(v j)=1 / 1+\mathrm{e}-\mathrm{avj}
$$

Training is performed multiple times over all input vectors in the training set. Weights may be updated incrementally after each input vector is presented or cumulatively after the training set in its entirety has been presented (one training epoch). This second approach, called batch learning, is an optimization of the back propagation algorithm designed to improve convergence by preventing individual input vectors from causing the computed error gradient to proceed in incorrect direction.

\section{Field Programmable Gate Array And Very High Hardware Description Language}

FPGAs consist of three basic blocks that are configurable logic blocks, in-out blocks and connection blocks. Logic blocks perform logic function. Connection blocks connect logic blocks with in-out blocks. These structures consist of routing channels and programmable switches. Routing process is effectively connection logic blocks exist different distance the others [6].

FPGAs are chosen for implementation ANNs with the following reason:

* They can be applied a wide range of logic gates starting with tens of thousands up to few millions gates.

* They can be reconfigured to change logic function while resident in the system.

* FPGAs have short design cycle that leads to fairly inexpensive logic design.

* FPGAs have parallelism in their nature. Thus, they have parallel computing environment and allows logic cycle design to work parallel.

* They have powerful design, programming and syntheses tools.

The architecture of ANNs must be specified with schematic or algorithmic at first step of FPGAs based system design. When ANNs based FPGAs system design specify the architecture of ANNs from a symbolic level. This level allows us using VHDL which stands for VHSIC (Very High Speed Integrated Circuit) Hardware Programming Language [7]. VHDL allows many levels of abstractions, and permits accurate description of electronic components ranging from simple logic gates to microprocessors. VHDL have tools needed for description and simulation which leads to a lower production cost.

\section{1 system architecture}

\section{Hardware Implementation}

The proposed system architecture is shown in fig.3 The proposed system working principles and detailed description is given below. The pressure sensor input is in analog nature this analog input is given to analog to digital converter. The signal conditioning circuit (ADC) convert analog signal into 8 bit digital signal. This 8 bit digital signal is given to the summing block then set the pressure selection value is given to the summing block. The summing block act as comparator, the comparator compares the input value and the reference value and give the difference value and it is denoted by en (error value). The error value is en is given to the ANN controller unit here the neural network is designed and trained to using the multilayer perceptron architecture and the back propagation algorithm. Then compute overall error and the output is given to digital to analog converter, then the digital to analog converter output is in current form so it is given to the current to voltage converter. Then the output is given to the modulation block, here pulse width modulation is done. The modulated signal given to pressure assembly control block. This is compute the overall error and measure the pressure value and function approximation. The pressure control driven circuit is used to activate pressure assembly control block. 


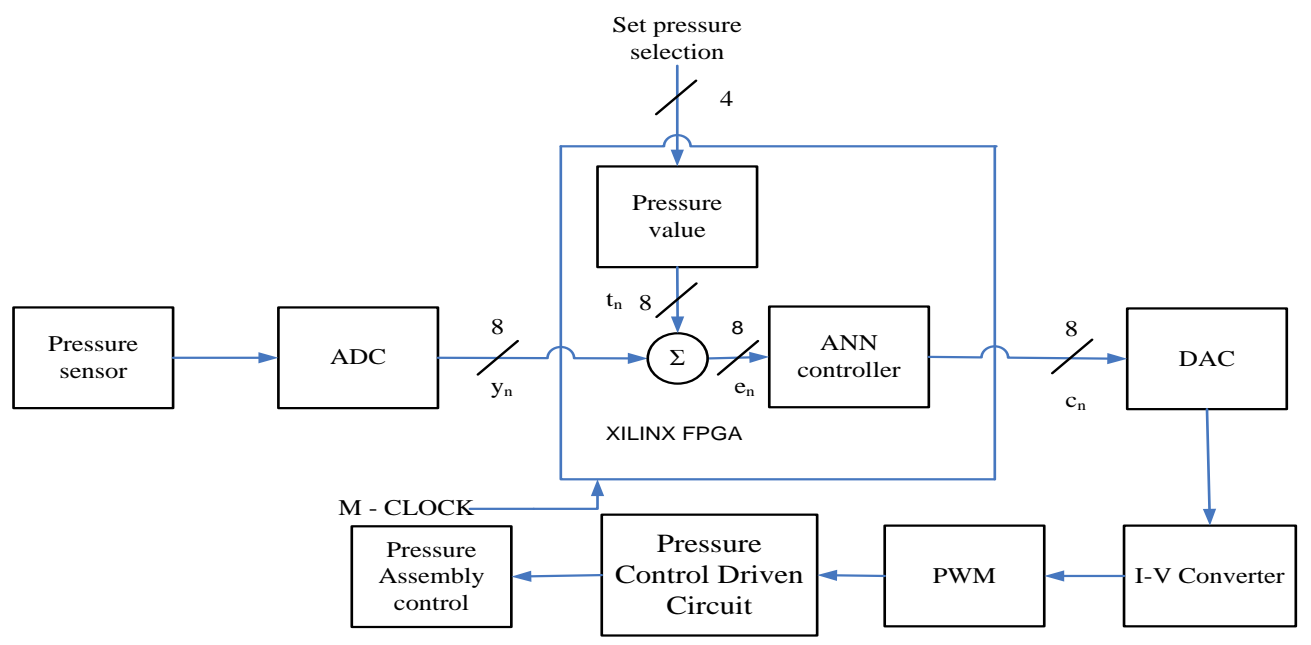

Fig.3. Block diagram of ANN implementation in FPGA

The pipelined architecture for ANN implementation in FPGA is shown in fig.4. Here set the pressure value and pressure sensor value is given to the $8 * 1$ multiplexer. The multiplexer output 8 bit digital value, and this is represented by th. This value is given to the 8 bit magnitude comparator. The comparator compares the pressure value register and the set pressure value, then the output represented by error(en). The error value is given to the input layer, then this value is given to the H1 MAC architecture here the input weight is multiplied by w1 register and this value is given to the $\mathrm{HI}$ neuron transfer function. and this value is given to the $\mathrm{H} 2 \mathrm{MAC}$ architectures, then the weight is multiplied by the w2 register and it is given to the $\mathrm{H} 2$ transfer function and the error propagates to hidden layer finally compute the overall error then the output matched with the set the value . and ANNs are trained to solve the pressure control function approximation.

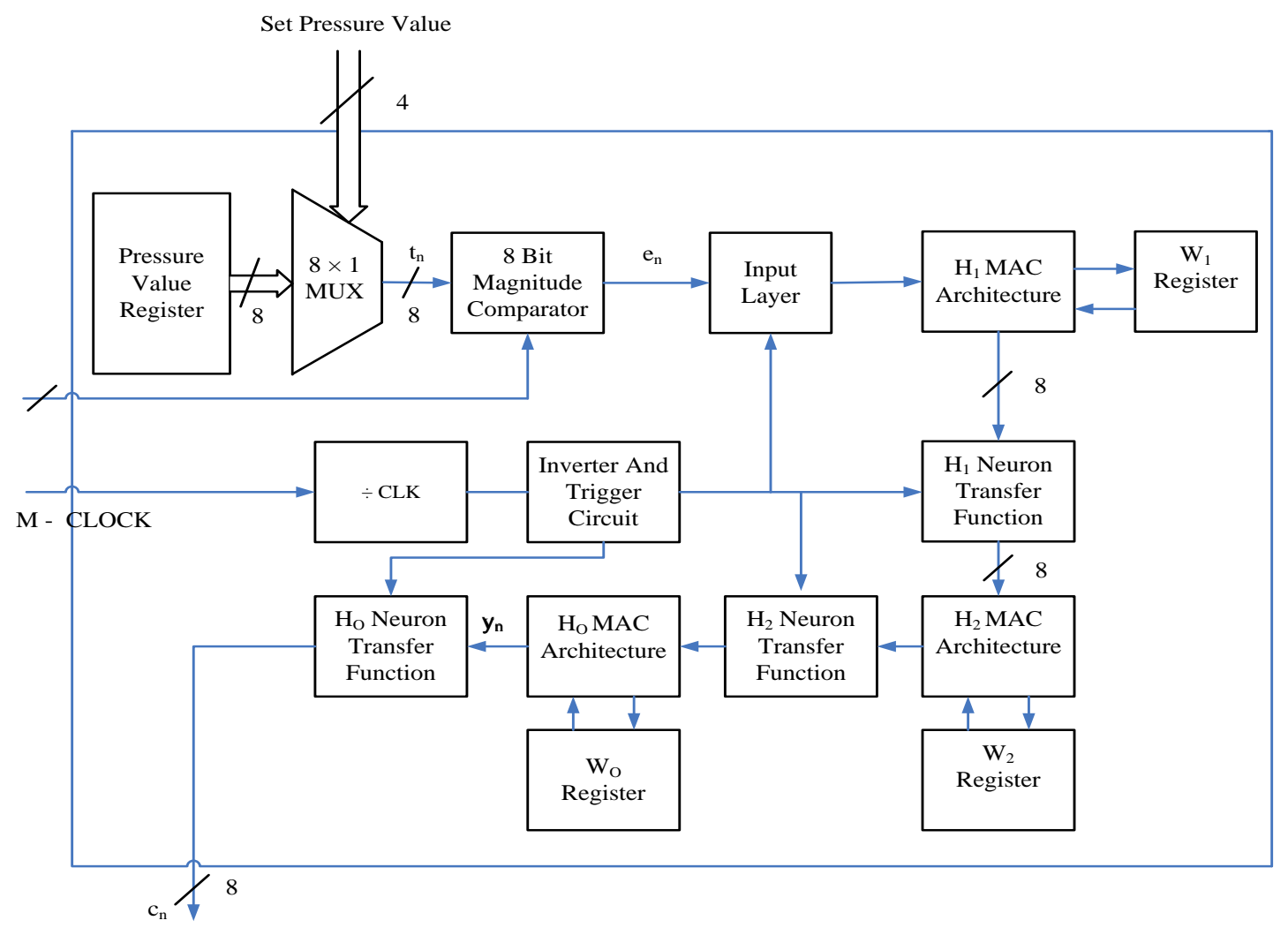




\section{Experimental Results}

The artificial neural network is designed and implement the pressure control application. The network implementation built using the MATLAB neural network tool box and find the minimum mean square error.

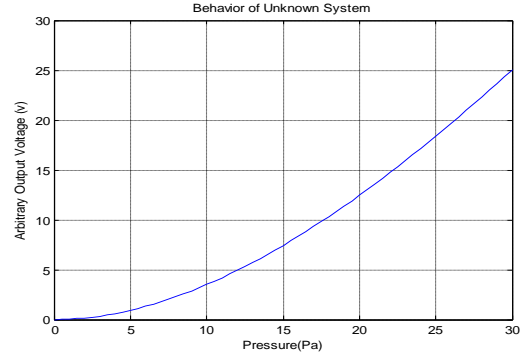

Fig.5. Behavior of unknown system

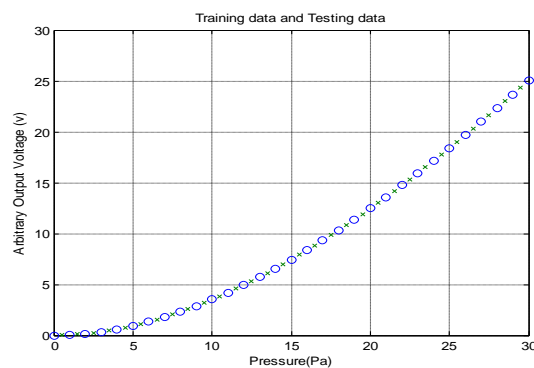

Fig.6. Training data and testing data

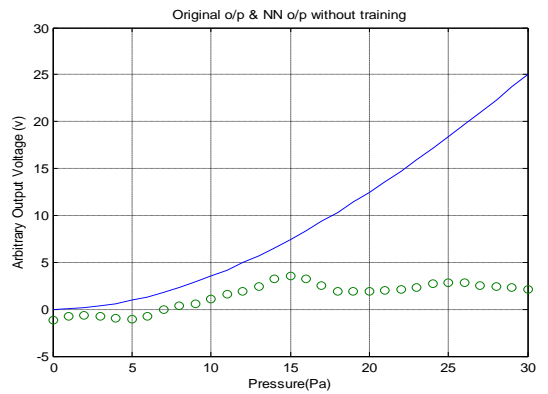

Fig 7. Original output and NN output without training

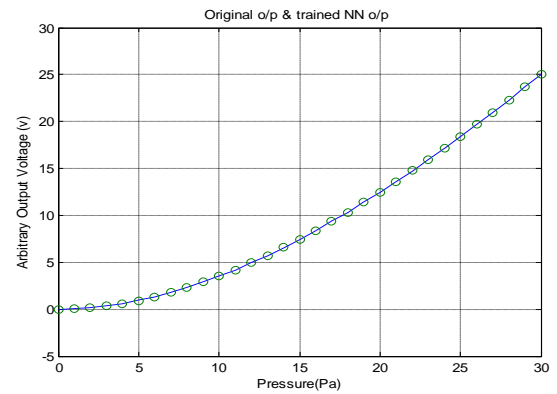

Fig.8 originl output and NN output

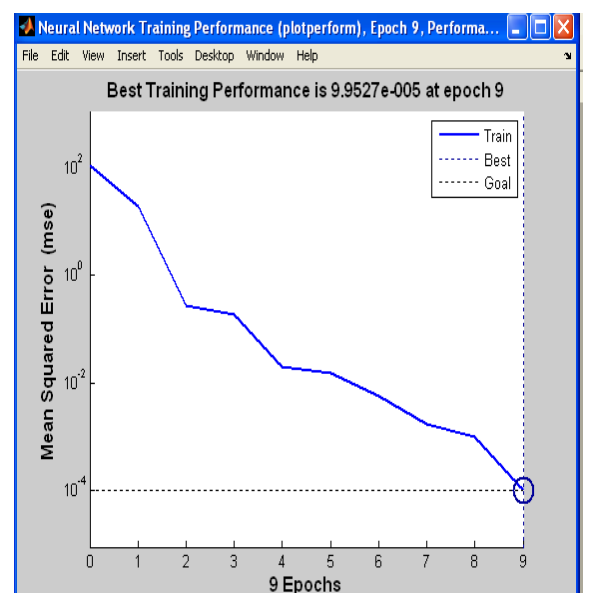

Fig.9 Neural network training performance

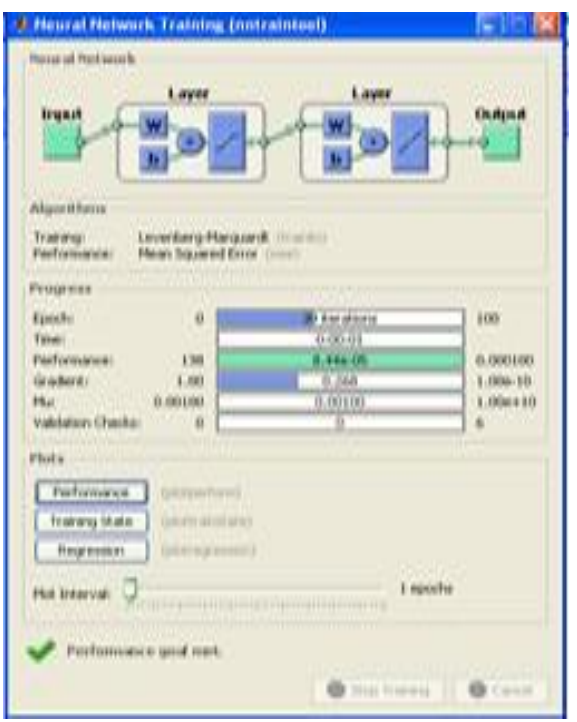

Fig.10 neural network toolbox

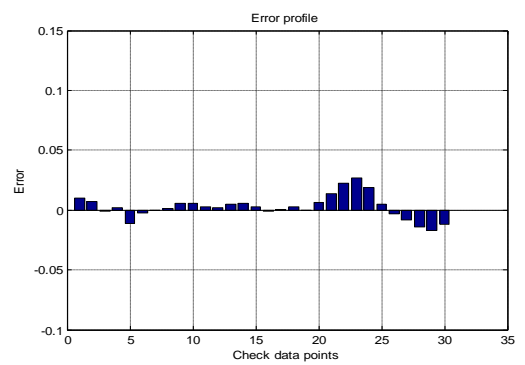

Fig.11 Error profile 


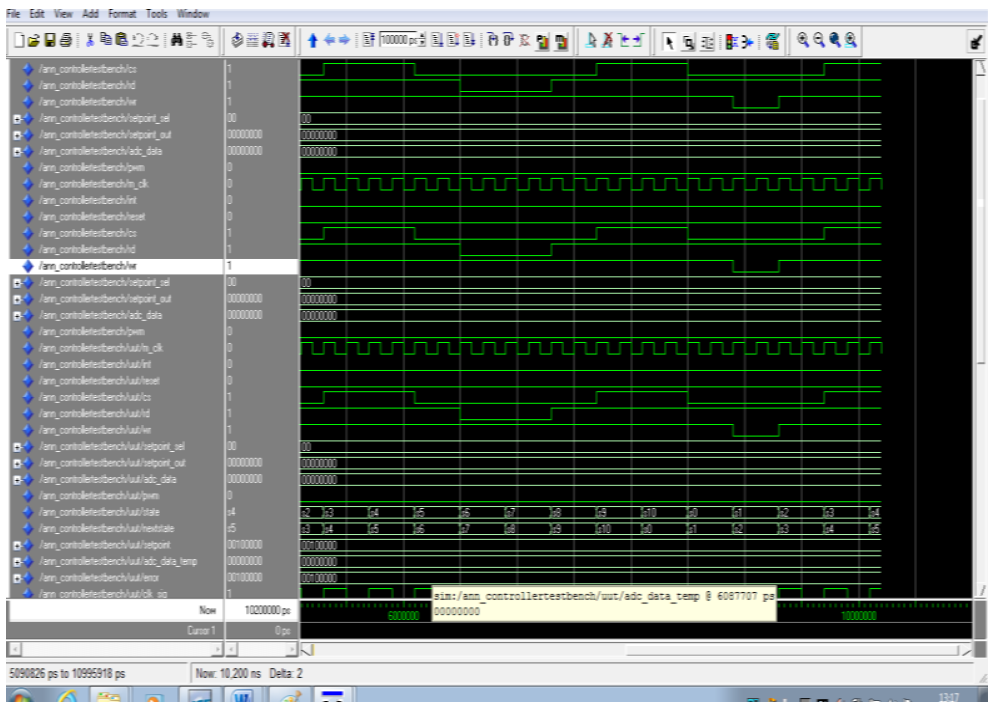

Fig.12 Simulation Result of Pressure Controller Controller

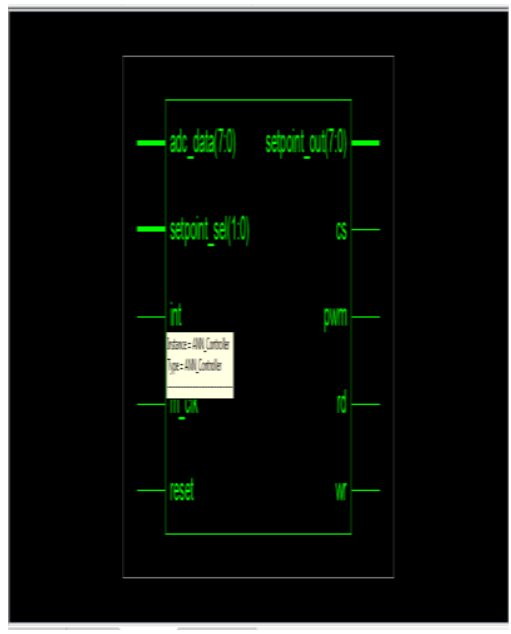

Fig.13 Schematic of Pressure

\section{Conclusion}

In this paper, presented the development and implementation of a pipelined FPGA-based architecture for feed-forward multilayer perception architecture with back propagation of learning algorithm. In general, it is shown that implementation of neural networks using FPGAs. The resultant neural networks are modular, compact, and efficient and the number of neurons, number of hidden layers and number of inputs are easily changed. However this study shows that FPGAs are versatile devices for implementing many different applications. The VHDL-FPGA combination is shown to be a very powerful embedded system design tool, with low cost, reliability. In conclusion, the ANNs are trained and testing the system using the function approximation for pressure control application. It showed that the system can reach best training performance i.e9.957at epoch 9 and the minimum mean square error is $10^{-4}$.These best training weights are implemented in FPGA board.

\section{References}

[1] M. Paliwal and U. A. Kumar, "Neural networks and statistical techniques: A review of applications," Expert Systems With Applications, vol. $\quad 36$, pp. 2-17, 2009

[2] R. Gadea, R. C. Palero, J. C. Boluda, and A. S. Cortes, "FPGA implementation of a pipelined on-line back propagation," J. VLSI SignalProcess., vol. 40, pp. 189-213, 2005.

[3] A. R. Ormondi and J. Rajapakse, FPGA Implementations of Neural Networks. New York: Springer, 2006

[4] M. Tommiska, "Efficient digital implementation of the sigmoid function for reprogrammable logic," in Proc. IEE Comput. Digital Tech., 2003, vol.150, pp. 403-4

[5] E. Sanchez, "FPGA implementation of an adaptable-size neural network," in Proc. Int. Conf. ANN, 1996, vol. 1112, pp. 383-388

[6] J. Li and D.Liang, "A survey of FPGA-based hardware implementation of ANNs," in Proc. Int. Conf. Neural Networks Brain, 2005, vol. 2, pp. 915-91

[7] R. Gadea, J. Cerda, F. Ballester, and A. Mocholi, "Artificial neural network implementation on a single FPGA of a pipeline on-line backpropagation," in Proc. Int. Symp.Syst. Synthesis, 2000, pp. 225-230

[8] P. Ferreiraa, P. Ribeiroa, A. Antunes, and F. M. Dias, "A high bit resolution FPGA implementation of a FNN with a new algorithm for the activation function," Neurocomputing, vol. 71, pp. 71-77, 2007.

[9] S.Tatikonda and P.Agarwal, "Field programmable gate array (FPGA) based neural network implementation of motion control and fault diagnosis of induction motor drive," in Proc. IEEE Conf. Ind. Tech., 2008, pp. 1-6.

[10] A. Mellit, H.Mekki, A. Messai, and H. Salhi, "FPGA-based implementation of an intelligent simulator for stand-alone photovoltaic system," Expert Systems With Applications, vol. 37, pp. 6036-6051, 2010 